\title{
Mechanism of Interaction of BMP and Insulin Signaling in C. elegans Development and Homeostasis
}

James F. Clark ${ }^{1,2}$, Emma J. Ciccarelli ${ }^{1,2}$, Gehan Ranepura ${ }^{1}$, Muhammad S. Hasan ${ }^{1}$, Alicia Meléndez ${ }^{1,2}$, Cathy Savage-Dunn ${ }^{1,2}$

'Biology Department, Queens College, CUNY, Flushing, New York 11367

${ }^{2}$ Ph.D. Program in Biology, The Graduate Center, City University of New York (CUNY), New York, New York 10016 


\section{Abstract}

A small number of peptide growth factor ligands are used repeatedly in development and homeostasis to drive programs of cell differentiation and function. Cells and tissues must integrate inputs from these diverse signals correctly, while failure to do so leads to pathology, reduced fitness, or death. Previous work using the nematode $C$. elegans identified an interaction between the bone morphogenetic protein (BMP) and insulin/IGF-1-like signaling (IIS) pathways in the regulation of lipid homeostasis. The molecular components required for this interaction, however, were not known. Here we report that INS-4, one of 40 insulin-like peptides (ILPS), is specifically regulated by BMP signaling to modulate fat accumulation. Furthermore, we find that the IIS transcription factor DAF-16/FoxO, but not SKN-1/Nrf, acts downstream of BMP signaling in lipid homeostasis. Interestingly, BMP activity alters sensitivity of these two transcription factors to IIS-promoted cytoplasmic retention in opposite ways. Finally, we probe the extent of BMP and IIS interactions by testing two additional IIS functions, dauer formation and autophagy induction. Coupled with our previous work and that of other groups, we conclude that BMP and IIS pathways have at least three modes of interaction: independent, epistatic, and antagonistic. The molecular interactions we identify provide new insight into mechanisms of signaling crosstalk and potential therapeutic targets for IIS-related pathologies such as diabetes and metabolic syndrome. 


\section{Introduction}

Cell signaling pathways are integral to normal development and homeostasis, and their misregulation is frequently associated with pathological conditions including cancer, cardiovascular disease, and metabolic syndrome. Cells and tissues have distinctive patterns of responsiveness to a variety of secreted peptide ligands. The correct integration of these signals allows for robust control of development and sensitive regulation of homeostatic processes. We have previously identified a regulatory interaction between bone morphogenetic protein (BMP) and insulin/IGF-1-like signaling (IIS) pathways in the regulation of lipid stores in the nematode Caenorhabditis elegans. BMPs are members of the Transforming Growth Factor beta (TGF $\beta$ ) family of peptide ligands. BMPs are conserved across animal phyla, and their actions are traditionally known for roles in development, growth, and differentiation (Shi and Massague 2003; Wu and Hill 2009). Our research, however, has explored the homeostatic effects of BMP signaling, using the powerful genetic tractability and imaging tools available in $C$. elegans to elucidate interactions between signaling pathways. In addition to our work, roles for TGF $\beta$ family members in regulating metabolic homeostasis in vertebrates have been elucidated recently (Wang et al. 2004; Fain et al. 2005; Sjoholm et al. 2006; Bottcher et al. 2009; Shen et al. 2009). BMPs in particular may play a role in insulin regulation and age-related insulin resistance (Chattopadhyay et al. 2017). Unlike BMP signaling, IIS is well-known to function in homeostatic processes. In mammals, IIS regulates glucose uptake and metabolic homeostasis (Anisimov and Bartke 2013). Disruptions to insulin balance, such as insulin resistance, can lead to multiple metabolic disorders and diseases, including obesity and type II diabetes. Studying these pathways in $C$. elegans is fruitful due to the high degree of conservation of the respective signaling pathways.

The BMP signaling pathway in $C$. elegans includes founding members of the Smad family of signal transducers, SMA-2, SMA-3, and SMA-4 (Savage et al. 1996; Savage-Dunn and Padgett 
2017). DBL-1, the C. elegans BMP2/4 homolog, plays a major role in body size regulation, male tail development, and mesodermal patterning (Suzuki et al. 1999; Foehr et al. 2006). Initial identification of several genes related to fat metabolism through microarray analysis in a $d b /-1$ mutant background (Liang et al. 2007) led to the hypothesis that DBL-1/BMP is a regulator of metabolism. We confirmed this hypothesis, showing that disruptions to DBL-1 signaling lead to reduced lipid stores and altered lipid droplet morphology. Additionally, we determined that this regulation of lipid storage by DBL-1/BMP is dependent on IIS activity (Clark et al. 2018).

In C. elegans, the IIS pathway uses a single insulin receptor DAF-2/InsR (Gottlieb and Ruvkun 1994; Kimura et al. 1997) in conjunction with 40 insulin-like peptides (ILPs) to regulate multiple homeostatic functions through the control of transcription factors, such as DAF-16/FoxO (Ogg et al. 1997; Paradis and Ruvkun 1998; Lin et al. 2001) and SKN-1/Nrf (Tullet et al. 2008). DAF-2/IIS has prolific effects on the development and homeostasis of the worm; disruptions to DAF-2/IIS lead to phenotypes in dauer formation, longevity, stress tolerance, innate immunity, germline maintenance, metabolism, and autophagy (Gems et al. 1998; Tissenbaum and Ruvkun 1998; Melendez et al. 2003; Jia et al. 2004; Brys et al. 2010; Michaelson et al. 2010; Kaplan and Baugh 2016).

In this study, we show an antagonistic effect between DBL-1/BMP and DAF-2/IIS in a daf-2 mutant background when considering dauer formation and autophagy induction. Together with our previously published data on lipid stores and body size (Clark et al. 2018), our results outline three potential modes of interaction between DBL-1/BMP and DAF-2/IIS: independent, as in body size; epistatic; as in lipid regulation; and antagonistic, as seen in dauer formation and autophagy induction. We observe that Smad signaling in the hypodermis (epidermis) regulates expression of ins-4, and that ins-4 mutants express a high-fat phenotype epistatic to that of sma-3/Smad, implicating INS-4 as the mediator for epistatic interaction. Additionally, we find 
that loss of $d b /-1$ alters the subcellular localization of DAF-16::GFP and SKN-1::GFP, and adjusts their sensitivity to DAF-2/IIS in opposite directions. Taken together, our data identify new methods of interaction that mesh the signaling outputs of two well-conserved pathways, BMP and IIS, in the regulation of homeostatic functions.

\section{Materials and Methods}

\section{Nematode strains and growth conditions}

C. elegans were maintained on $E$. coli (DA837) at $15^{\circ} \mathrm{C}, 20^{\circ} \mathrm{C}$, and $25^{\circ} \mathrm{C}$ as specified. The wildtype strain used in this study was N2. The strains used in this study are as follows: LT121 dbl-1(wk70), CB678 lon-2(e678), CS24 sma-3(wk30), CB1370 daf-2(e1370), RB2544 ins-4(ok3534), HT1693 unc-119(ed3);wwEx63, DA2123 adls2122, MAH14 daf-2(e1370);adls2122, TJ356 zls356[daf-16::gfp], LD1 ldls7[skn-1::gfp], CS683 daf-2(e1370)sma-3(wk30), CS681 daf-2(e1370);Ion-2(e678). Crosses were used to obtain: CS663 sma-3(wk30);ins-4(ok3534), CS633 sma-3(wk30);wwEx63, CS661 daf-2(e1370);dbl-1(wk70);adls2122, CS634 daf-2(e1370);lon-2(e678);adls2122, CS626 dbl-1(wk70);adls2122, CS627 lon-2(e678);adls2122, CS685 dbl-1(wk70);zls356, and CS679 $d b l-1(w k 70) ; / d l s 7$.

\section{INS-4p::GFP Expression}

Animals were grown at $20^{\circ} \mathrm{C}$ and imaged as day 1 adults. Images were taken on a Leica Microsystems Confocal microscope using a 20X objective. Camera settings were maintained identically between all samples and trials. Compound images were produced from compiling Zstack images. $\mathrm{n}>10$ per strain repeated in triplicate. 


\section{Oil Red O staining}

Protocol adapted from (O'Rourke et al. 2009). Animals were collected at the L4 stage in PCR tube caps and washed three times in PBS. Worms were then fixed for 1 hour in $60 \%$ isopropanol while rocking at room temperature. The isopropanol was removed and worms were stained overnight with $60 \%$ Oil Red O solution while rocking at room temperature. Oil Red O was removed and the worms were washed once with PBS w/ .01\% Triton and left in PBS. Worms were mounted and imaged using an AxioCam MRc camera with AxioVision software. Images were taken using a 40X objective. Oil Red O stock solution was made with $0.25 \mathrm{~g}$ Oil Red $\mathrm{O}$ in $50 \mathrm{~mL}$ isopropanol. Intensity of the post-pharyngeal intestine was determined using ImageJ software. Pixel intensity was measured in the green color channel of the images. Three measurements using a 50px by 50px area were taken for each worm with background intensity subtracted for each individual picture. Statistical comparisons (two-way ANOVA, one-way ANOVA with post-hoc Tukey's multiple comparisons test, and unpaired t-test) were performed using GraphPad Prism 7 software. For each experiment, $n>20$ per strain repeated in triplicate.

\section{Dauer formation assay}

Five to ten $\mathrm{L} 4$ animals were placed per plate to lay eggs overnight at $15^{\circ} \mathrm{C}$. Adults and $\mathrm{L} 1 \mathrm{~s}$ were removed on the subsequent day with $\mathrm{M9}$. Plates with remaining eggs were then placed at either $20^{\circ} \mathrm{C}$ or $25^{\circ} \mathrm{C}$ to develop. Animals were then observed 48 hours later; dauer-like larvae were tallied versus L4s and adults. Statistical comparisons (unpaired t test and two-way ANOVA) were performed using GraphPad Prism 7 software. $n>50$ per strain at each temperature, repeated in triplicate.

\section{GFP::LGG-1 autophagy assay}

Autophagy was assayed via the formation of GFP punctae as described in (Palmisano and Meléndez 2016). Animals were grown at $20^{\circ} \mathrm{C}$ and imaged at the L3 larval stage; dauer larvae 
were not imaged or quantified. GFP::LGG-1 punctae were counted in the seam cells of the hypodermis. Images were taken using a Zeiss ApoTome with AxioVision software and a 100X objective. Exposure times were kept consistent across strains within each experiment.

Statistical comparisons (unpaired t test and one-way ANOVA) were performed using GraphPad Prism 7 software. $n=15$ per strain, repeated in duplicate.

\section{DAF-16::GFP and SKN-1::GFP Localization Assays}

RNAi induction and feeding were carried out as described in (Kamath et al. 2000; Liang et al. 2013). For DAF-16::GFP, L4 animals were placed on EZ worm plates supplemented with $1 \mathrm{mM}$ IPTG and $50 \mathrm{mg} / \mathrm{ml}$ carbenicillin overnight. Adults were moved to new RNAi plates and allowed to lay eggs for 4-6 hours to synchronize the progeny. Animals were then allowed to develop at $15^{\circ} \mathrm{C}$ until the L3 larval stage followed by a shift to $20^{\circ} \mathrm{C}$ to bypass the dauer induction of daf- 2 knockdown. L4 animals were assayed for GFP localization. L4440 was used as the empty vector control. Images were taken using a Zeiss ApoTome with AxioVision software and a 40X objective. Exposure times were kept consistent across strains within each experiment. GFP localization was categorized as none, low, medium, or high. $\mathrm{n}>20$ per strain, repeated in duplicate. For SKN-1::GFP, L4 animals were placed on EZ worm plates supplemented with $1 \mathrm{mM}$ IPTG and $50 \mathrm{mg} / \mathrm{ml}$ carbenicillin seeded with HT115 E. coli. Animals were allowed to lay eggs overnight. Adults and L1 larvae were removed the following day with M9. Remaining eggs were synchronized with a 4 to 6 hour hatch, with the L1s being transferred to new RNAi plates. Animals were then allowed to develop at $15^{\circ} \mathrm{C}$ until the $\mathrm{L} 3$ larval stage followed by a shift to $25^{\circ} \mathrm{C}$ to bypass the dauer induction of daf- 2 knockdown. Animals were left at $25^{\circ} \mathrm{C}$ overnight and then assayed for GFP localization. L4440 was used as the empty vector control. Images were taken using a Zeiss ApoTome with AxioVision software and a 20X objective. Exposure times were kept consistent across strains within each experiment. GFP localization was 
categorized as low, medium, or high. $n>40$ per strain, repeated in duplicate. Statistical comparisons (chi-square test) were performed using GraphPad Prism 7 software.

\section{$\underline{\text { Results }}$}

\section{ins-4 Regulation in the Hypodermis by SMA-3/Smad}

In previous work, we showed that SMA-3/Smad activity in the hypodermis (epidermis) regulates fat accumulation in the $C$. elegans intestine, and that SMA-3/Smad regulation of fat stores depends on the DAF-2 Insulin Receptor (InsR) (Clark et al. 2018). We therefore hypothesized that SMA-3/Smad may regulate expression of an insulin-like peptide (ILP) in the hypodermis, which then modulates DAF-2/InsR activity. To identify such an ILP, we considered two transcriptional targets previously identified by microarray analysis, ins-4 and ins-7 (Liang et al. 2007). Of these, ins-4 is reported to be expressed in hypodermis and neurons, whereas ins-7 expression has been observed in the intestine and in neurons (Murphy et al. 2007; Ritter et al. 2013). To observe INS-4 regulation, we obtained an ins-4p::gfp reporter and crossed it into a sma-3 mutant background. In wild-type animals carrying the ins-4p::gfp reporter, GFP was visible in neurons while only faint GFP expression was observed in hypodermis. However, in sma-3;ins-4p::gfp animals, GFP expression was starkly increased in the hypodermis, indicating an increase in ins-4 expression (Figure 1A). This observation indicates that DBL-1/BMP signaling normally down-regulates the expression of ins-4, corroborating the data from the microarray analysis. We conclude that SMA-3/Smad regulates expression of ins- 4 in the hypodermis.

\section{Loss of ins-4 Blocks the Low-Fat Phenotype of sma-3 Mutants}

If INS-4 acts as the mediator between Smad signaling in the hypodermis and DAF-2/InsR signaling in the intestine, it may be required for normal lipid accumulation. To date, single mutants for $C$. elegans ILP genes have not displayed mutant phenotypes, due to redundancy 
between the genes (Ritter et al. 2013; Zheng et al. 2018a). Nevertheless, we measured lipid accumulation in ins-4 mutant animals via Oil Red O staining. We observed an increase of $\sim 20 \%$ in the ins-4 mutants compared to wild-type $(\mathrm{p}=0.0016)$ (Figure $1 \mathrm{~B}, \mathrm{C})$, similar to the increase seen in daf-2 mutants (Kimura et al. 1997; Clark et al. 2018). We therefore conclude that INS-4 represses fat accumulation and is required for normal lipid stores. As previously published, we saw a significant decrease in sma-3 animals by $\sim 42 \%(p<0.0001)$. To test the proposed epistatic interaction between sma-3 and ins-4, we generated a sma-3;ins-4 double mutant and determined its phenotype. sma-3;ins-4 double mutants have a high fat accumulation phenotype compared to wild type $(p<0.0001)$ (Figure 1B,C), consistent with INS-4 acting downstream of SMA-3. Interestingly, we also saw a significant increase in staining of the sma-3;ins-4 double mutants compared with ins-4 single mutants (Figure 1B,C), an observation to which we will return in the Discussion. These results indicate that the loss of ins- 4 is sufficient to alter significantly the level of lipids in the intestine. Additionally, the loss of ins-4 reverses the loss of lipids in a sma-3 mutant background, instead resulting in an increased level of lipids compared to wild type. These findings implicate INS-4 as the target of action for DBL-1/BMP signaling to regulate lipid storage through DAF-2/IIS.

\section{DBL-1/BMP Signaling Promotes Dauer Arrest in a DAF-2/IIS-Sensitized Background}

Since we discovered that DBL-1/BMP and DAF-2/IIS pathways interact to regulate lipid accumulation, we sought to characterize the relationship between the DBL-1/BMP and DAF-2/IIS pathways in other biological contexts. During development, animals exposed to harsh environmental conditions prior to the third larval stage can activate the stress-adaptive and growth-arrested dauer larval program. IIS is one of the primary regulators of the dauer decision, and daf-2(e1370) mutants have a temperature-sensitive dauer-constitutive phenotype. We investigated the degree of dauer formation in animals containing mutations in BMP and IIS pathways at the restrictive temperature, $25^{\circ} \mathrm{C}$, and the semi-permissive temperature, $20^{\circ} \mathrm{C}$. 
Normally, the DBL-1/BMP pathway has no effect on dauer, as $d b /$-1 single mutants, and other mutants in the pathway, have no observable dauer phenotype at any temperature. As expected, when we placed sma-3 or lon-2 mutants at $25^{\circ} \mathrm{C}$, we observed no dauer arrest phenotype, as in our wild-type control. When daf-2 single mutants were placed at the restrictive temperature, we observed dauer arrest in $98.4 \pm 0.7 \%$ of the progeny, similar to previously published data. This phenotype was also observed in the daf-2;sma-3 and daf-2;/on-2 double mutants, with $99.5 \pm 0.6 \%$ and $97.6 \pm 1.7 \%$ dauer formation, respectively.

When animals were grown at the semi-permissive temperature, $20^{\circ} \mathrm{C}$, however, $\mathrm{DBL}-1 / \mathrm{BMP}$ signaling had an observable impact on arrested animals in the daf-2 mutant background. Again, the sma-3 and lon-2 mutants and wild type had no dauer induction at $20^{\circ} \mathrm{C}$. daf- 2 single mutants exhibited the dauer arrest phenotype in $54.5 \pm 6.3 \%$ of the progeny at the semi-permissive temperature. The daf-2;sma-3 double mutants, in contrast, exhibited the dauer arrest phenotype in only $23.9 \pm 4.6 \%$ of the animals, while the daf-2;/on-2 double mutants exhibited the dauer arrest phenotype in $73.4 \pm 7.3 \%$ of the animals (Table 1). Two-way ANOVA indicates a significant $(p=0.0006)$ interaction between the two pathways. This result indicates that $D B L-1 / B M P$ signaling has a significant effect on the dauer arrest phenotype in the DAF-2/IIS-sensitive background. DBL-1/BMP signaling appears to modulate dauer formation in a dose-dependent manner, where reduced signaling, as in daf-2; $d b /-1$ double mutants, reduces the occurrence of dauer-like animals, while increased signaling, as in daf-2;lon-2 mutants, increases the occurrence of dauer-like animals.

\section{DBL-1/BMP Signaling Enhances Autophagic Induction in a DAF-2/IIS-Sensitized Background}

Autophagy is a cellular process involved in the recycling of cellular debris and other material, and is vital for the maintained health of an organism (Melendez and Levine 2009). As a known 
regulator of autophagy, DAF-2/IIS acts to suppress the induction of autophagosome formation, with daf-2 mutants exhibiting increased levels of autophagy. Additionally, autophagy is required for proper dauer development in daf-2 mutants (Meléndez et al. 2003). DBL-1/BMP signaling has also been shown to inhibit autophagy, as mutants in the BMP pathway elevate clearance of aggregates of SQST-1/p62 autophagy receptor induced by proteotoxic stress (Guo et al. 2014). We reproduced the effect of BMP signaling on SQST-1::GFP accumulation following RNAi inhibition of SMA-3/Smad (S1 Fig).

We also measured autophagosome levels through the use of GFP::LGG-1, an autophagosome reporter (Palmisano and Meléndez 2016). LGG-1/LC3 is a protein involved in the formation, elongation, and fusion of autophagosomes (Meléndez et al. 2003). Normally localized in a diffuse cytosolic pattern, when autophagic conditions are induced, GFP::LGG-1 becomes localized to pre-autophagosomal structures and autophagosomes. The localization of GFP::LGG-1 has been previously observed in hypodermal seam cells. The seam cells form a row of cells that run the length of the hypodermis on each lateral side of the animal, and undergo remodelling in the development of dauer animals. Thus, seam cells were observed due to their size and ease in imaging. daf-2 mutants are known to show increased levels of autophagy when grown at the semi-permissive and restrictive temperatures, $20^{\circ} \mathrm{C}$ and $25^{\circ} \mathrm{C}$ respectively. To determine if DBL-1/BMP signaling would behave similarly in the regulation of autophagy as it did with dauer formation, we grew the animals at $20^{\circ} \mathrm{C}$. We observed an average of $5.51 \pm 2.42$ punctae per seam cell in daf- 2 single mutants at $20^{\circ} \mathrm{C}$. In daf-2;dbl-1 double mutants, the average was decreased to $3.85 \pm 2.20$ ( $p<0.0001)$, while in daf-2;/on-2 double mutants, the average was increased to $6.90 \pm 2.75(p<0.0001)$ (Figure 2). Therefore, DBL-1/BMP activity antagonizes the effect of DAF-2/IIS in the induction of autophagy. 
The observations presented above, combined with previous work from our lab (Clark et al. 2018), identifies three modes of interaction between DBL-1/BMP signaling and DAF-2/IIS: independent, epistatic, and antagonistic. DBL-1/BMP and DAF-2/IIS regulate body size independently, as double mutants in the two pathways were observed to have additive effects (Clark et al. 2018). Epistatic effects were observed in the regulation of lipids with the high-fat phenotype of daf-2 mutants completely masking the low-fat phenotype of sma-3 in daf-2;sma-3 double mutants. Lastly, the data observed in this study reveals a third relationship between the two pathways; DBL-1/BMP signaling appears to antagonistically affect DAF-2/IIS phenotypes. However, these effects are only observed in a DAF-2/IIS-compromised background. To elucidate further the context-dependent mechanisms by which DBL-1/BMP interacts with DAF-2/IIS, we explored interactions with transcription factors downstream of DAF-2.

\section{Loss of DBL-1/BMP Signaling Alters Localization of Transcription Factors Downstream of DAF-2/IIS}

When DAF-2/IIS is activated, both DAF-16/FoxO and SKN-1/Nrf are phosphorylated and excluded from the nucleus; however, upon down-regulation of DAF-2/IIS, both transcription factors are shuttled into the nucleus (Ogg et al. 1997; Tullet et al. 2008). To observe any alterations to this translocation, we crossed DAF-16::GFP and SKN-1::GFP transgenes into a dbl-1 mutant background and treated animals with daf-2 RNAi. Localization of the two transcription factors was observed in the intestinal nuclei due to their size and prominence.

We categorized nuclear localization of the GFP fusion proteins in these backgrounds as none (cytoplasmic only), low (diffuse in both cytoplasm and nucleus), medium (faint nuclear localization) or high (strong nuclear localization). In a wild-type background, animals treated with the empty vector, L4440, showed DAF-16::GFP fluorescence diffusely or weakly nuclear, with a small percentage showing only cytoplasmic localization (Figure 3). As expected, treatment with 
daf-2 RNAi caused nuclear accumulation of DAF-16::GFP in all animals $(p=0.0017)$. In $d b /-1$ mutant animals on control RNAi plates, there was no significant difference in the level of nuclear localization compared with the wild-type controls (Figure 3). Upon reduction of $d b /$-1 and daf-2 together, however, intensity of nuclear DAF-16::GFP became higher than in daf-2(RNAi) animals alone $(p<0.0001)$ (Figure 3$)$. We conclude that loss of $d b /-1$ increases the responsiveness of the DAF-16::GFP reporter to DAF-2 activity.

In a wild-type background, animals treated with the empty vector, L4440, exhibited mainly diffuse expression of SKN-1::GFP, with very few animals containing nuclear localization. Upon treatment with daf-2 RNAi, the majority of animals showed a significant increase in nuclear SKN-1::GFP, as expected ( $p<0.0001)$. When treated with L4440, $d b /-1$ mutants exhibited an increased level of nuclear localization compared to the wild-type control $(p=0.0073)$, but less than that of wild type treated with daf-2 RNAi $(p<0.0001)$. Interestingly, upon treatment with daf-2 RNAi, localization of SKN-1::GFP in $d b /-1$ mutants was not significantly different from those treated with the empty vector $(p=0.8694)$ (Figure $4 A, B)$. This outcome contrasts with the results with DAF-16::GFP. Instead, the responsiveness of the SKN-1::GFP reporter to the reduction of DAF-2/IIS was reduced in the $d b /-1$ mutant background.

\section{Effects of IIS Transcription Factors on $d b /-1$ Low-Fat Phenotype}

We sought to determine whether one or both of these transcription factors mediate regulation of fat storage downstream of DBL-1/BMP and DAF-2/InsR. The strains expressing tagged functional DAF-16/FoxO or SKN-1/Nrf contain multicopy arrays that may cause an increase in the expression levels of these transcription factors. We measured fat accumulation using Oil Red O staining in L4 stage animals. In the wild-type background, neither daf-16::gfp nor skn-1::gfp have increased fat stores (Figure 5). Next, we examined $d b /-1$ mutant animals. As expected, $d b /-1$ mutants have low fat stores. daf-16::gfp expression, but not skn-1::gfp 
expression, is capable of suppressing the low-fat phenotype of $d b /-1$ mutants (observed in two out of three trials). This result suggests that SKN-1 does not mediate the effect of DBL-1 on fat accumulation.

\section{Discussion}

We previously showed that DBL-1/BMP signaling and DAF-2/IIS interact to regulate lipid stores in C. elegans (Clark et al. 2018). In this study, we identify additional interactions by presenting evidence that the DBL-1/BMP signaling pathway exhibits an antagonistic effect on dauer formation and autophagy induction in a DAF-2/InsR sensitized mutant background. We show that loss of BMP signaling activity causes a reduction in the rate of both dauer formation and autophagy, in daf-2 mutants at $20^{\circ} \mathrm{C}$. Conversely, increased BMP signaling causes an increase in the rates of these phenotypes. These interactions are only revealed in a DAF-2-compromised background, distinguishing them from the epistatic relationship of BMP and IIS in lipid regulation and the independent functions of BMP and IIS in body size regulation and reproductive aging (Luo et al. 2010; Clark et al. 2018). Our findings implicate coordination between BMP and IIS pathways in mutliple aspects of development and homeostasis, an interpretation that is supported by additional evidence in both $C$. elegans and other systems. For example, DBL-1/BMP interacts with IIS in C. elegans L1 arrest. In C. elegans, embryos that hatch without food enter an L1 arrest in which cell divisions are blocked. The arrested somatic development of different cell lineages has been shown to be dependent on DBL-1/BMP interaction with IIS. In mesodermal lineages, the cell division arrest is defective in daf-16 mutants, and DBL-1/BMP activity is required for the L1 arrest defective phenotype of daf-16 (Kaplan et al. 2015). In neuronal lineages, DBL-1/BMP acts upstream to promote IIS activity in a DAF-16-independent role, through reduction in the expression of ins-3 and ins-4 in neurons (Zheng et al. 2018b). In addition, DBL-1/BMP signaling interacts with DAF-16/FoxO function to induce germline tumor formation (Qi et al. 2017). In vertebrates, tissue culture studies have shown that BMP2 and 
BMP6 regulate insulin sensitivity in adipose cells (Schreiber et al. 2017). In mice, BMP4 regulates insulin sensitivity of adipose tissue (Qian et al 2013.). Furthermore, BMP7 regulates expression of insulin signaling components in C3H10T1/2 cells, leading to fat browning (Zhang et al. 2010). Numerous additional instances of interactions between TGF $\beta /$ activin signaling and IIS also exist, emphasizing the widespread deployment of this signaling axis.

This work has uncovered specific components of the IIS pathway that mediate the effects of DBL-1/BMP on lipid accumulation. Two ILPS, INS-4 and INS-7, are transcriptionally repressed by DBL-1/BMP signaling (Liang et al. 2007). Using an ins-4p::gfp reporter, we now show that ins-4 expression is highly and specifically increased in the hypodermis in a sma-3 mutant background, indicating that Smad signaling regulates ins-4 expression in the hypodermis. Additionally, we show that ins-4 mutants have increased levels of lipids, demonstrating that, in spite of any potential redundancy between ILPS, INS-4 is critically required for regulation of lipid storage. Consistent with our analysis, overexpression of ins-4 is sufficient to lower fat levels (Zheng et al. 2018a). Finally, we determined that the ins-4 phenotype is epistatic to the sma-3 low-fat phenotype, since sma-3;ins-4 mutants exhibit a high-fat phenotype. These data provide evidence that DBL-1 activates Smad signaling in the hypodermis, down-regulating the expression of INS-4, to reduce DAF-2/IIS in the intestine, in turn, maintaining proper lipid stores (Figure 6A). The other ILP gene we identified as a transcriptional target of DBL-1, ins-7, has no effect on fat accumulation when overexpressed (Zheng et al. 2018a). Since ins-7 is a known transcriptional target of IIS in the intestine (Murphy et al. 2007), its upregulation in $d b /-1$ mutants may be a secondary consequence of increased IIS activity. We also interrogated the effects of downstream IIS transcription factors DAF-16/FoxO and SKN-1/Nrf on DBL-1-mediated fat regulation. Since these transcription factors act in opposition to the other IIS components, we did not analyze loss-of-function mutants but rather overexpression from multi-copy transgenes. 
Our results are consistent with DAF-16/FoxO, but not SKN-1/Nrf, mediating the effect of DBL-1 on fat accumulation (Figure 6B).

IIS transcription factors DAF-16/FoxO and SKN-1/Nrf are regulated, in part, at the level of subcellular localization. DAF-2/IIS activity promotes cytoplasmic retention of DAF-16/FoxO and SKN-1/Nrf, inhibiting their functions as transcription factors. In this study, we find that DBL-1/BMP activity alters the sensitivity of DAF-16/FoxO and SKN-1/Nrf to DAF-2/IIS in opposite ways. In $d b /-1$ mutants, SKN-1::GFP localization is not significantly altered by daf-2 RNAi (Figure 2), indicating that DBL-1/BMP is required for full responsiveness of SKN-1/Nrf to DAF-2/IIS. For DAF-16::GFP, we see the opposite effect on sensitvity to DAF-2/IIS. In $d b /-1$ mutants, DAF-16::GFP is less nuclear than control and upon treatment with daf-2(RNAi) it becomes intensely nuclear (Figure 3). The elevated nuclear DAF-16 in combined BMP and IIS knockdown may contribute to the higher fat accumulation we observed in ins-4;sma-3 double mutants (Figure 1). If DBL-1/BMP signaling caused a simple reduction in DAF-2/IIS activity, we would have expected both transcription factors to respond similarly, with increased cytoplasmic localization in a dbl-1 mutant and possibly equivalent nuclear localization upon daf-2 RNAi in both wild-type and $d b /-1$ backgrounds. Two possible mechanisms for the observed differences between DAF-16 and SKN-1, which are not mutually exclusive, are that INS-4 activation of DAF-2/InsR differentially affects the downstream transcription factors, or that DBL-1/BMP signaling has an additional direct influence on localization independent of IIS.

While specific domains of expression can provide fine-tuned control of ligand-receptor combinations in an organism, individual ligands can also produce alternate responses through a single receptor. In mammals, the Fibroblast Growth Factor (FGF) signaling family consists of 18 ligands (FGFs) and four tyrosine kinase receptors (FGFRs) (Ornitz and Itoh 2015). In the FGF signaling family, there exist examples of two ligands with overlapping expression patterns that 
elicite different responses from the same receptor. Both FGF7 and FGF10 exhibit roles in the branching morphogeneis of the lacrimal and submandibular glands in mice using ex vivo organ cultures. These two ligands both bind to FGFR2b, however, FGF7 induces a proliferative response while FGF10 induces a migratory response, switching morphogenesis between branching and elongation respectively (Steinberg et al. 2005; Makarenkova et al. 2009). The mechanism thought to underly this switch is through the differential phosphorylation of a specific tyrosine residue. Binding of FGF10 to FGFR2b results in the phosphorylation of residue Y734, which recruits a complex involved in endocytic recycling, while binding of FGF7 does not (Francavilla et al. 2013). The exact mechanism by which the two ligands result in differential phosphorylation is unknown, however structural evidence suggests that binding of the ligands may induce two different confirmations of the intracellular domains (Bae and Schlessinger 2010). While this mechanism has not been studied in the context of DAF-2/IIS in $C$. elegans, it does not preclude the possibility that this mechanism may be shared between the two tyrosine kinase receptor families. While the 40 insulin-like peptides present in $C$. elegans can be classified as either agonists or antagonists of DAF-2, differential effects within a classification could be attributed to a similar mechanism.

Altered subcellular localization of DAF-16/FoxO provides a mechanism for the demonstrated inhibitory interaction between BMP and IIS in lipid metabolism. In addition to the interactions already discussed, Qi et al. identified a cooperative interaction between DBL-1/BMP signaling and DAF-16/FoxO that does not occur at the level of subcellular localization (Qi et al. 2017). They find that DAF-16/FoxO functions nonautonomously in the hypodermis to induce germline tumor formation, and that this function depends on DBL-1/BMP signaling. This phenotype is independent of nuclear localization of DAF-16/FoxO; rather the Smads SMA-2 and SMA-3 were shown to bind DAF-16 directly and presumably promote its transcriptional activity (Qi et al. 2017). Taken together, these analyses exemplify the interconnected, polymodal nature of BMP 
and IIS pathway interactions in the regulation of organismal function. The homeostatic regulation of cellular processes, such as metabolism, are vital to the well-being of an organism and must be flexible enough to coordinate a variety of inputs and outputs. In mammals, the complexity of tissues involved in such crosstalk creates a challenge for precise analysis of these interactions. Using $C$. elegans, we have developed a model to understand the relationship of two highly conserved pathways, BMP and IIS, in the intact organism.

\section{Acknowledgments}

We thank Michael Meade and Shoshana Reich for the construction of CS663 and CS633, respectively. We thank Nick Palmisano for input on autophagy assays. This work was supported in part by National Institutes of Health R15GM112147 to CSD, and by 2R15GM102846-02 to AM. Some strains were provided by the CGC, which is funded by NIH Office of Research Infrastructure Programs (P40OD010440). This work was carried out in partial fulfillment of the requirements for the Ph.D. degree from the Graduate Center of City University of New York (JFC and EJC). 


\section{$\underline{\text { References }}$}

Anisimov VN, Bartke A. 2013. The key role of growth hormone-insulin-IGF-1 signaling in aging and cancer. Critical reviews in oncology/hematology 87: 201-223.

Bae JH, Schlessinger J. 2010. Asymmetric Tyrosine Kinase Arrangements in Activation or Autophosphorylation of Receptor Tyrosin Kinases. Mol Cells 29: 443-448.

Bottcher Y, Unbehauen H, Kloting N, Ruschke K, Korner A, Schleinitz D, Tonjes A, Enigk B, Wolf $\mathrm{S}$, Dietrich $\mathrm{K}$ et al. 2009. Adipose tissue expression and genetic variants of the bone morphogenetic protein receptor 1A gene (BMPR1A) are associated with human obesity. Diabetes 58: 2119-2128.

Brys K, Castelein N, Matthijssens F, Vanfleteren JR, Braeckman BP. 2010. Disruption of insulin signalling preserves bioenergetic competence of mitochondria in ageing Caenorhabditis elegans. BMC Biol 8: 91.

Chattopadhyay T, Singh RR, Gupta S, Surolia A. 2017. Bone morphogenetic protein-7 (BMP-7) augments insulin sensitivity in mice with type II diabetes mellitus by potentiating PI3K/AKT pathway. BioFactors 43: 195-209.

Clark JF, Meade M, Ranepura G, Hall DH, Savage-Dunn C. 2018. Caenorhabditis elegans DBL1/BMP Regulates Lipid Accumulation via Interaction with Insulin Signaling. G3 (Bethesda) 8: 343-351.

Fain JN, Tichansky DS, Madan AK. 2005. Transforming growth factor b1 release by human adipose tissue is enhanced in obesity. Metabolism: clinical and experimental 54: 15461551.

Foehr ML, Lindy AS, Fairbank RC, Amin NM, Xu M, Yanowitz J, Fire AZ, Liu J. 2006. An antagonistic role for the $C$. elegans Schnurri homolog SMA-9 in modulating TGFb signaling during mesodermal patterning. Development 133: 2887-2896.

Francavilla C, Rigbolt KT, Emdal KB, Carraro G, Vernet E, Bekker-Jensen DB, Streicher W, Wikstrom M, Sundstrom M, Bellusci $S$ et al. 2013. Functional proteomics defines the molecular switch underlying FGF receptor trafficking and cellular outputs. Mol Cell 51: 707-722.

Gems D, Sutton AJ, Sundermeyer ML, Albert PS, King KV, Edgley ML, Larsen PL, Riddle DL. 1998. Two pleiotropic classes of daf-2 mutation affect larval arrest, adult behavior, reproduction and longevity in Caenorhabditis elegans. Genetics 150: 129-155.

Gottlieb S, Ruvkun G. 1994. daf-2, daf-16 and daf-23: genetically interacting genes controlling Dauer formation in Caenorhabditis elegans. Genetics 137: 107-120.

Guo B, Huang X, Zhang P, Qi L, Liang Q, Zhang X, Huang J, Fang B, Hou W, Han J et al. 2014. Genome-wide screen identifies signaling pathways that regulate autophagy during Caenorhabditis elegans development. EMBO reports 15: 705-713.

Jia K, Chen D, Riddle DL. 2004. The TOR pathway interacts with the insulin signaling pathway to regulate C. elegans larval development, metabolism and life span. Development 131: 3897-3906.

Kamath RS, Martinez-Campos M, Zipperlen P, Faser AG, Ahringer J. 2000. Effectiveness of specific RNA-mediated interference through ingested double-stranded RNA in Caenorhabditis elegans. Genome Biology 2: research0002.0001-0002.0010.

Kaplan RE, Baugh LR. 2016. L1 arrest, daf-16/FoxO and nonautonomous control of postembryonic development. Worm 5: e1175196.

Kaplan RE, Chen Y, Moore BT, Jordan JM, Maxwell CS, Schindler AJ, Baugh LR. 2015. dbl1/TGF-beta and daf-12/NHR Signaling Mediate Cell-Nonautonomous Effects of daf16/FOXO on Starvation-Induced Developmental Arrest. PLoS Genet 11: e1005731.

Kimura KD, Tissenbaum HA, Liu Y, Ruvkun G. 1997. daf-2, an insulin receptor-like gene that regulates longevity and diapause in Caenorhabditis elegans. Science 277: 942-946. 
Liang J, Xiong S, Savage-Dunn C. 2013. Using RNA-mediated interference feeding strategy to screen for genes involved in body size regulation in the nematode $C$. elegans. Journal of visualized experiments : JoVE.

Liang J, Yu L, Yin J, Savage-Dunn C. 2007. Transcriptional repressor and activator activities of SMA-9 contribute differentially to BMP-related signaling outputs. Developmental Blology 305: 714-725.

Lin K, Hsin H, Libina N, Kenyon C. 2001. Regulation of the Caenorhabditis elegans longevity protein DAF-16 by insulin/IGF-1 and germline signaling. Nat Genet 28: 139-145.

Luo S, Kleemann GA, Ashraf JM, Shaw WM, Murphy CT. 2010. TGF-b and insulin signaling regulate reproductive aging via oocyte and germline quality maintenance. Cell 143: 299312.

Makarenkova HP, Hoffman MP, Beenken A, Eliseenkova AV, Meech R, Tsau C, Patel VN, Lang RA, Mohammadi M. 2009. Differential interactions of FGFs with heparan sulfate control gradient formation and branching morphogenesis. Sci Signal 2: ra55.

Melendez A, Levine B. 2009. Autophagy in C. elegans. WormBook: doi/10.1895/wormbook.1891.1147.1891.

Melendez A, Talloczy Z, Seaman M, Eskelinen EL, Hall DH, Levine B. 2003. Autophagy genes are essential for dauer development and life-span extension in C. elegans. Science 301: 1387-1391.

Meléndez A, Talloczy Z, Seaman M, Eskelinen EL, Hall DH, Levine B. 2003. Autophagy genes are essential for dauer development and life-span extension in C. elegans. Science 301: 1387-1391.

Michaelson D, Korta DZ, Capua Y, Hubbard EJ. 2010. Insulin signaling promotes germline proliferation in C. elegans. Development 137: 671-680.

Murphy CT, Lee SJ, Kenyon C. 2007. Tissue entrainment by feedback regulation of insulin gene expression in the endoderm of Caenorhabditis elegans. Proceedings of the National Academy of Sciences of the United States of America 104: 19046-19050.

O'Rourke EJ, Soukas AA, Carr CE, Ruvkun G. 2009. C. elegans major fats are stored in vesicles distinct from lysosome-related organelles. Cell metabolism 10: 430-435.

Ogg S, Paradis S, Gottlieb S, Patterson GI, Lee L, Tissenbaum HA, Ruvkun G. 1997. The Fork head transcription factor DAF-16 transduces insulin-like metabolic and longevity signals in C. elegans. Nature 389: 994-999.

Ornitz DM, Itoh N. 2015. The Fibroblast Growth Factor signaling pathway. Wiley Interdiscip Rev Dev Biol 4: 215-266.

Palmisano NJ, Meléndez A. 2016. Detecting Autophagy in Caenorhabditis elegans Using GFP::LGG-1 as an Autophagy Marker. Cold Spring Harb Protoc 1: pdb.prot086496.

Paradis S, Ruvkun G. 1998. Caenorhabditis elegans Akt/PKB transduces insulin receptor-like signals from AGE-1 PI3 kinase to the DAF-16 transcription factor. Genes Dev 12: 24882498.

Qi W, Yan Y, Pfeifer D, Donner VGE, Wang Y, Maier W, Baumeister R. 2017. C. elegans DAF16/FOXO interacts with TGF-b/BMP signaling to induce germline tumor formation via mTORC1 activation. PLoS genetics 13: e1006801.

Qian SW, Tang Y, Li X, Liu Y, Zhang YY, Huang HY, Xue RD, Yu HY, Guo L, Gao HD et al. 2013. BMP4-mediated brown fat-like changes in white adipose tissue alter glucose and energy homeostasis. Proceedings of the National Academy of Sciences of the United States of America 110: E798-807.

Ritter AD, Shen Y, Fuxman Bass J, Jeyaraj S, Deplancke B, Mukhopadhyay A, Xu J, Driscoll M, Tissenbaum HA, Walhout AJ. 2013. Complex expression dynamics and robustness in $C$. elegans insulin networks. Genome research 23: 954-965.

Savage-Dunn C, Padgett RW. 2017. The TGF-b Family in Caenorhabditis elegans. Cold Spring Harbor perspectives in biology 9 . 
Savage C, Das P, Finelli AL, Townsend SR, Sun CY, Baird SE, Padgett RW. 1996. Caenorhabditis elegans genes sma-2, sma-3, and sma-4 define a conserved family of transforming growth factor beta pathway components. Proceedings of the National Academy of Sciences of the United States of America 93: 790-794.

Schreiber I, Dorpholz G, Ott CE, Kragesteen B, Schanze N, Lee CT, Kohrle J, Mundlos S, Ruschke K, Knaus P. 2017. BMPs as new insulin sensitizers: enhanced glucose uptake in mature 3T3-L1 adipocytes via PPARgamma and GLUT4 upregulation. Scientific reports 7: 17192.

Shen JJ, Huang L, Li L, Jorgez C, Matzuk MM, Brown CW. 2009. Deficiency of growth differentiation factor 3 protects against diet-induced obesity by selectively acting on white adipose. Molecular endocrinology 23: 113-123.

Shi Y, Massague J. 2003. Mechanisms of TGF-beta signaling from cell membrane to the nucleus. Cell 113: 685-700.

Sjoholm K, Palming J, Lystig TC, Jennische E, Woodruff TK, Carlsson B, Carlsson LM. 2006. The expression of inhibin beta $B$ is high in human adipocytes, reduced by weight loss, and correlates to factors implicated in metabolic disease. Biochemical and biophysical research communications 344: 1308-1314.

Steinberg Z, Myers C, Heim VM, Lathrop CA, Rebustini IT, Stewart JS, Larsen M, Hoffman MP. 2005. FGFR2b signaling regulates ex vivo submandibular gland epithelial cell proliferation and branching morphogenesis. Development 132: 1223-1234.

Suzuki Y, Yandell MD, Roy PJ, Krishna S, Savage-Dunn C, Ross RM, Padgett RW, Wood WB. 1999. A BMP homolog acts as a dose-dependent regulator of body size and male tail patterning in Caenorhabditis elegans. Development 126: 241-250.

Tissenbaum HA, Ruvkun G. 1998. An insulin-like signaling pathway affects both longevity and reproduction in Caenorhabditis elegans. Genetics 148: 703-717.

Tullet JM, Hertweck M, An JH, Baker J, Hwang JY, Liu S, Oliveira RP, Baumeister R, Blackwell TK. 2008. Direct inhibition of the longevity-promoting factor SKN-1 by insulin-like signaling in C. elegans. Cell 132: 1025-1038.

Wang W, Yang Y, Meng Y, Shi Y. 2004. GDF-3 is an adipogenic cytokine under high fat dietary condition. Biochemical and biophysical research communications 321: 1024-1031.

Wu MY, Hill CS. 2009. Tgf-beta superfamily signaling in embryonic development and homeostasis. Developmental cell 16: 329-343.

Zhang H, Schulz TJ, Espinoza DO, Huang TL, Emanuelli B, Kristiansen K, Tseng YH. 2010. Cross talk between insulin and bone morphogenetic protein signaling systems in brown adipogenesis. Molecular and cellular biology 30: 4224-4233.

Zheng S, Chiu H, Boudreau J, Papanicolaou T, Bendena W, Chin-Sang I. 2018a. A functional study of all 40 Caenorhabditis elegans insulin-like peptides. The Journal of biological chemistry 293: 16912-16922.

Zheng S, Qu Z, Zanetti M, Lam B, Chin-Sang I. 2018b. C. elegans PTEN and AMPK block neuroblast divisions by inhibiting a BMP-insulin-PP2A-MAPK pathway. Development 145. 


\section{Percentage dauer arrest by daf-2;sma-3 mutants}

\begin{tabular}{lccccc}
\hline \multirow{2}{*}{ Strain } & \multicolumn{2}{c}{$\mathbf{2 0 ^ { \circ } \mathbf { C }}$} & & \multicolumn{2}{c}{$\mathbf{2 5 ^ { \circ } \mathbf { C }}$} \\
\cline { 2 - 3 } \cline { 5 - 6 } N2 & \% Arrested & $\mathbf{N}$ & & \% Arrested & $\mathbf{N}$ \\
sma-3(wk30) & 0 & 239 & & 0 & 228 \\
lon-2(e678) & 0 & 243 & & 0 & 178 \\
daf-2(e1370) & $54.5 \pm 4.5$ & 273 & & $98.4 \pm 0.5$ & 236 \\
daf-2(e1370);sma-3(wk30) & $23.9 \pm 3.3$ & 200 & & $99.6 \pm 0.4$ & 221 \\
daf-2(e1370);Ion-2(e678) & $73.5 \pm 5.2$ & 317 & & $97.6 \pm 1.2$ & 199 \\
\hline
\end{tabular}

Table 1. DBL-1 Pathway Modulates Dauer Constitutive Phenotype of daf-2 Mutants at

$20^{\circ} \mathrm{C}$. In a WT background, DBL-1 signaling exerts no visible effect on dauer induction at both $20^{\circ} \mathrm{C}$ and $25^{\circ} \mathrm{C}$. However, in a DAF-2-sensitized background, DBL-1 signaling promotes dauer arrest at $20^{\circ} \mathrm{C}$. This effect is lost when observed at $25^{\circ} \mathrm{C}$, where the loss of DAF-2 signaling is most potent. Values given are percent arrested dauer-like larva of total progeny \pm SEM. 
A
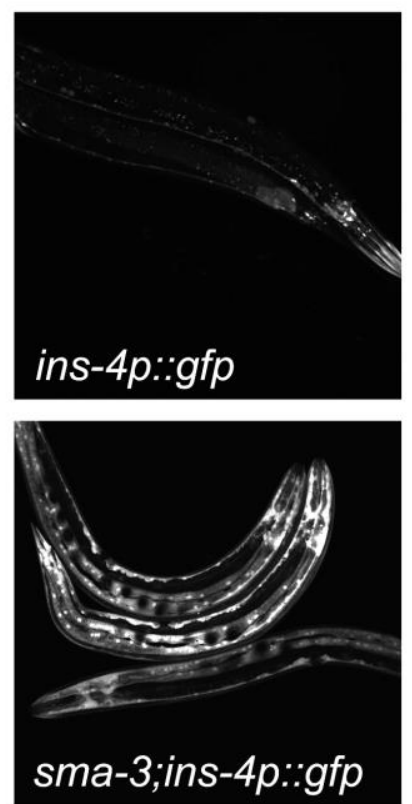

B

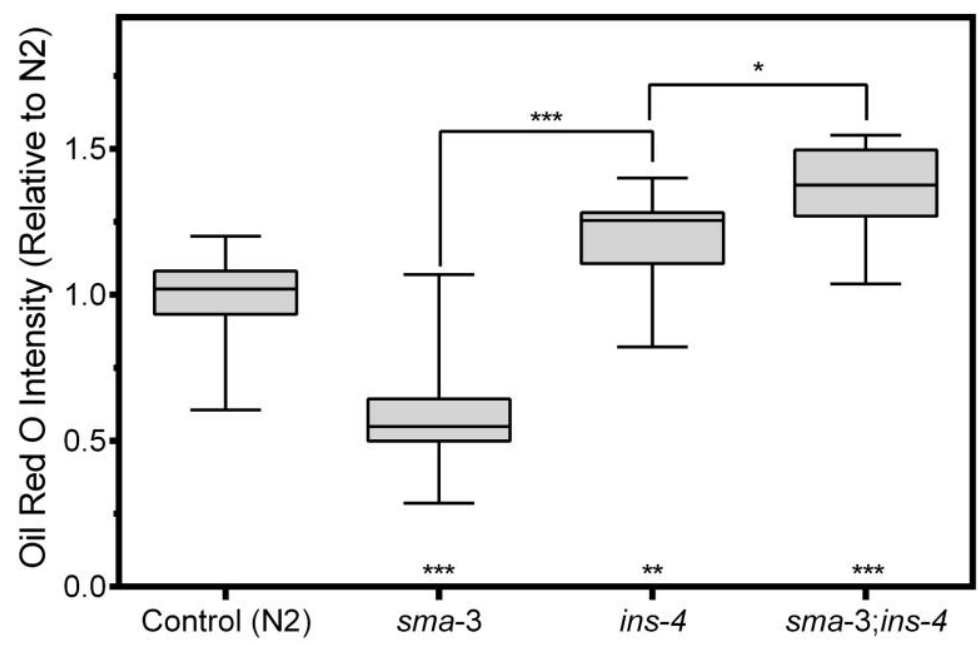

C

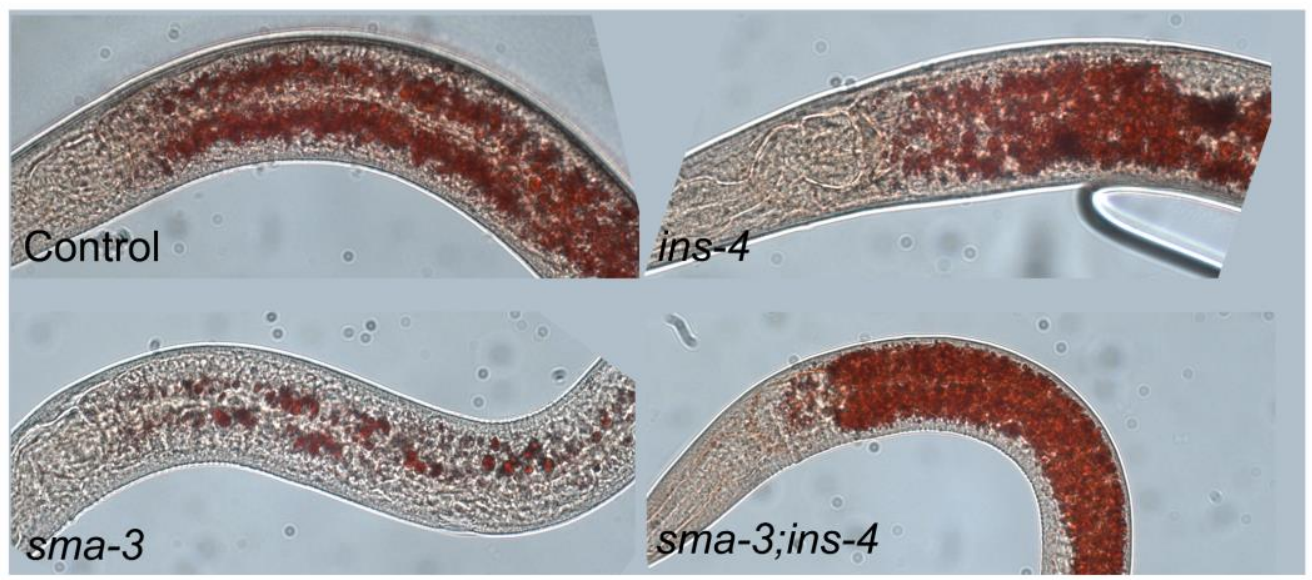

Figure 1. INS-4 acts downstream of SMA-3 to regulate lipid metabolism. A) Images of animals expressing ins-4p::GFP. Control animals displayed ins-4p::GFP in head neurons, with faint expression in the hypodermis. Upon loss of sma-3, ins-4p::GFP expression is significantly increased in the hypodermis. Images taken at 200X, camera settings were kept constant between all strains. B) Loss of ins-4 results in an increase in intestinal lipid stores via Oil Red O staining, this increase is epistatic to the low-fat phenotype of sma-3 mutants. Animals were grown at $20^{\circ} \mathrm{C}$ and stained at the L4 larval stage. Quantification was done for equivalent regions of the intestine just posterior to the pharynx. C) Images of L4 animals stained with Oil Red O 
bioRxiv preprint doi: https://doi.org/10.1101/777805; this version posted September 23, 2019. The copyright holder for this preprint (which was not certified by peer review) is the author/funder, who has granted bioRxiv a license to display the preprint in perpetuity. It is made available under aCC-BY-NC 4.0 International license.

taken at 400X. Anterior is to the left. For all graphs, asterisks across the bottom denote significance compared to Control, n.s. not significant, ${ }^{\star} p$ value $<0.05,{ }^{* *} p$ value $<0.001$, boxes denote the $2^{\text {nd }}$ and $3^{\text {rd }}$ quartiles, whiskers denote min and max values. 


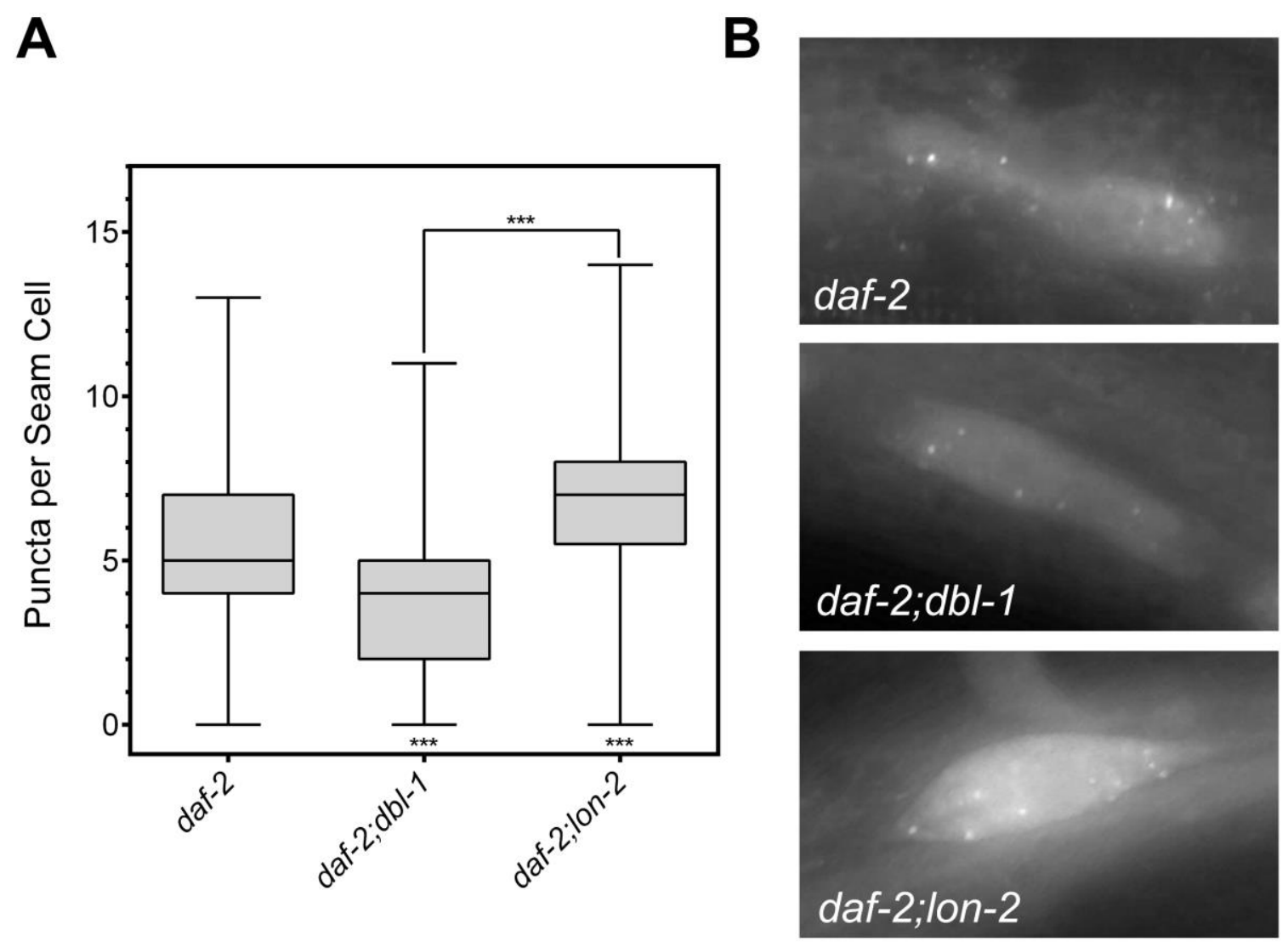

Figure 2. DBL-1 signaling promotes autophagy in a DAF-2-sensitized background.

A) When autophagy is induced, via a reduction in DAF-2 signaling, DBL-1 signaling promotes autophagy, as daf-2(e1370);dbl-1(wk70) mutants show a reduction in GFP puncta, while daf-2(e1370);Ion-2(e678) mutants show an increase in puncta. B) Images of GFP::LGG-1 localization in seam cells of DBL-1 pathway mutants under autophagic induction via daf-2 mutant background at $630 \mathrm{X}$. All animals were grown at $20^{\circ} \mathrm{C}$ and imaged at the $\mathrm{L} 3$ larval stage. ${ }^{\star * \star} p$ value $<0.001$, boxes denote $2^{\text {nd }}$ and $3^{\text {rd }}$ quartiles, whiskers denote min and max values. 


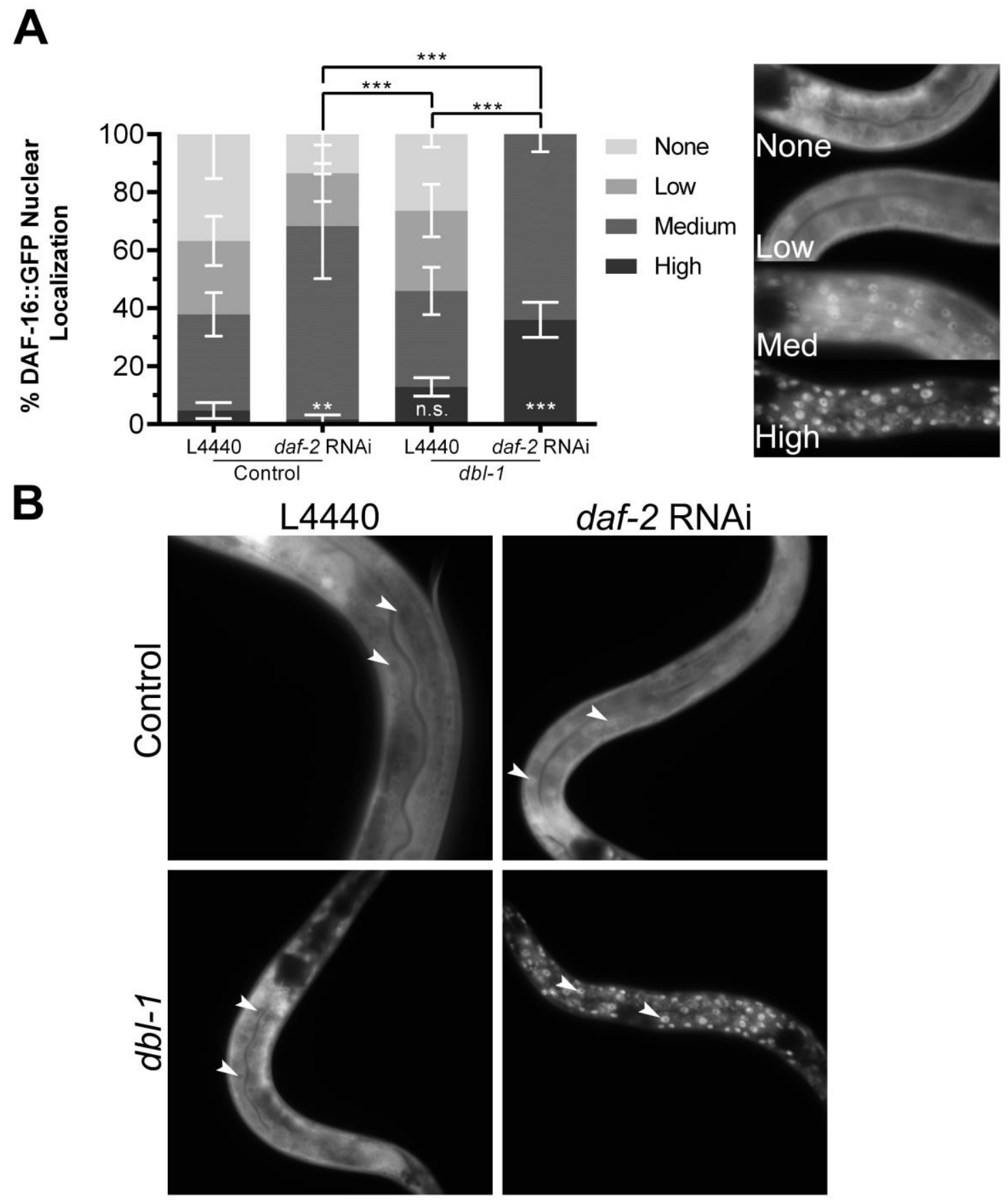

Figure 3. DAF-16 localization is altered in a $d b l-1$ mutant background.

Control animals treated with empty vector exhibit some nuclear localization of DAF-16::GFP, upon treatment with daf-2 RNAi, DAF-16::GFP nuclear localization increases. In dbl-1 mutants, DAF-16::GFP nuclear localization is not significantly changed compared to the control under 
empty vector treatment. Upon treatment with daf-2 RNAi, a higher level of nuclear localization is observed as compared to control animals treated with daf-2 RNAi. Asterisks across the bottom represent significance compared to Control, n.s. not significant, ${ }^{\star *} p<0.01,{ }^{\star \star *} p<0.001$, error bars represent SEM. B) Images of animals expressing DAF-16::GFP taken at 400X. White arrows indicate examples of intestinal nuclei. Camera settings were identical between all strains. 

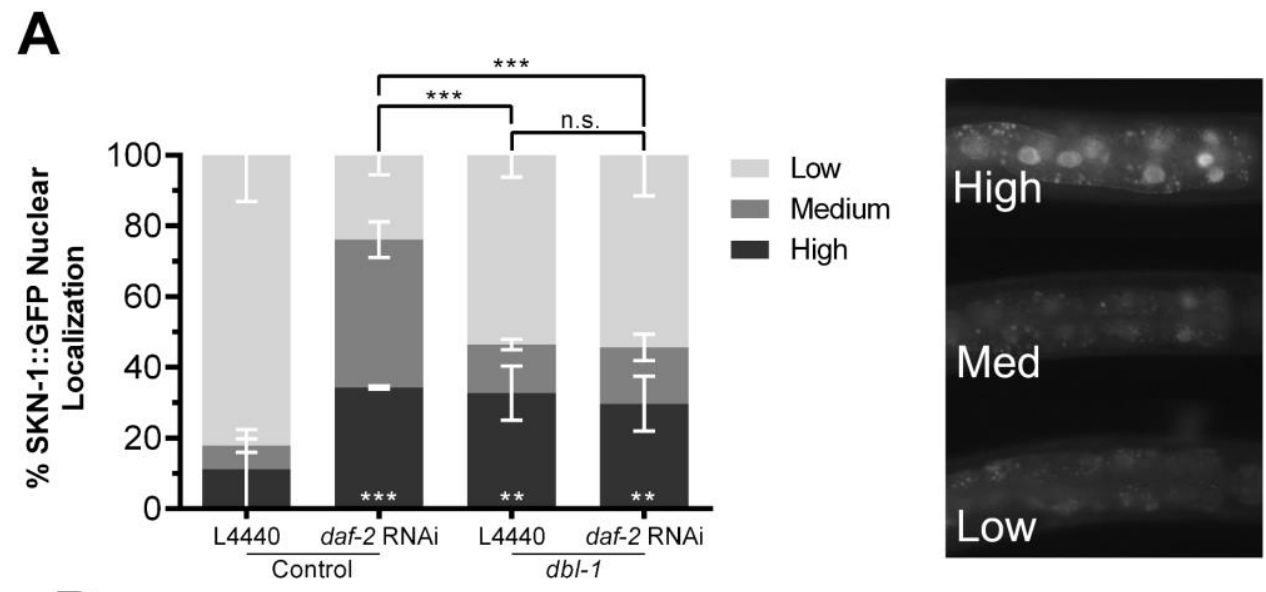

B
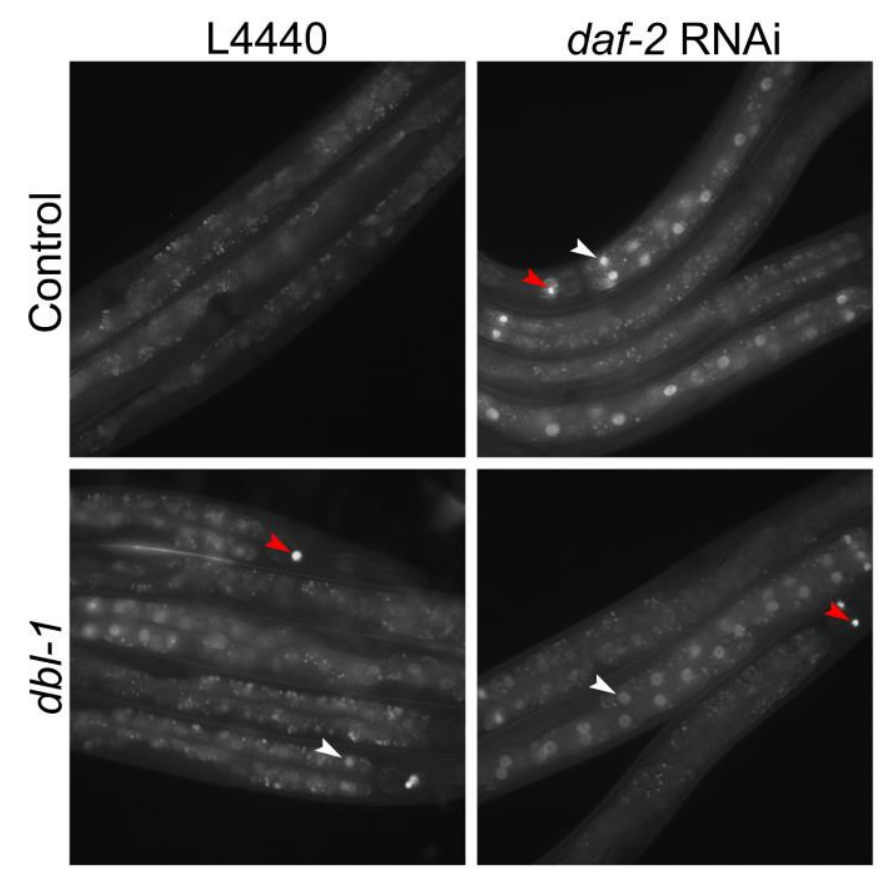

Figure 4. SKN-1::GFP localization is altered in $d b /-1$ mutants regardless of DAF-2/IIS. A)

Control animals treated with empty vector exhibit very little nuclear localization of SKN-1::GFP, upon treatment with daf-2 RNAi, SKN-1::GFP nuclear localization drastically increases. In $d b /-1$ mutants, SKN-1::GFP nuclear localization is slightly increased compared to the control under empty vector treatment, upon treatment with daf-2 RNAi, no change in nuclear localization is observed. Nuclear localization was graded as high, medium, or low, with an example of each ranking shown. Asterisks across the bottom represent significance compared to Control, n.s. not significant, ${ }^{* *} p<0.01,{ }^{* * *} p<0.001$, error bars represent SEM. B) Images of animals expressing 
bioRxiv preprint doi: https://doi.org/10.1101/777805; this version posted September 23, 2019. The copyright holder for this preprint (which was not certified by peer review) is the author/funder, who has granted bioRxiv a license to display the preprint in perpetuity. It is made available under aCC-BY-NC 4.0 International license.

SKN-1::GFP taken at 200X. White arrows indicate examples of nuclei, red arrows indicate

SKN-1::GFP expression in ASI neurons. Camera settings were identical between all strains. 


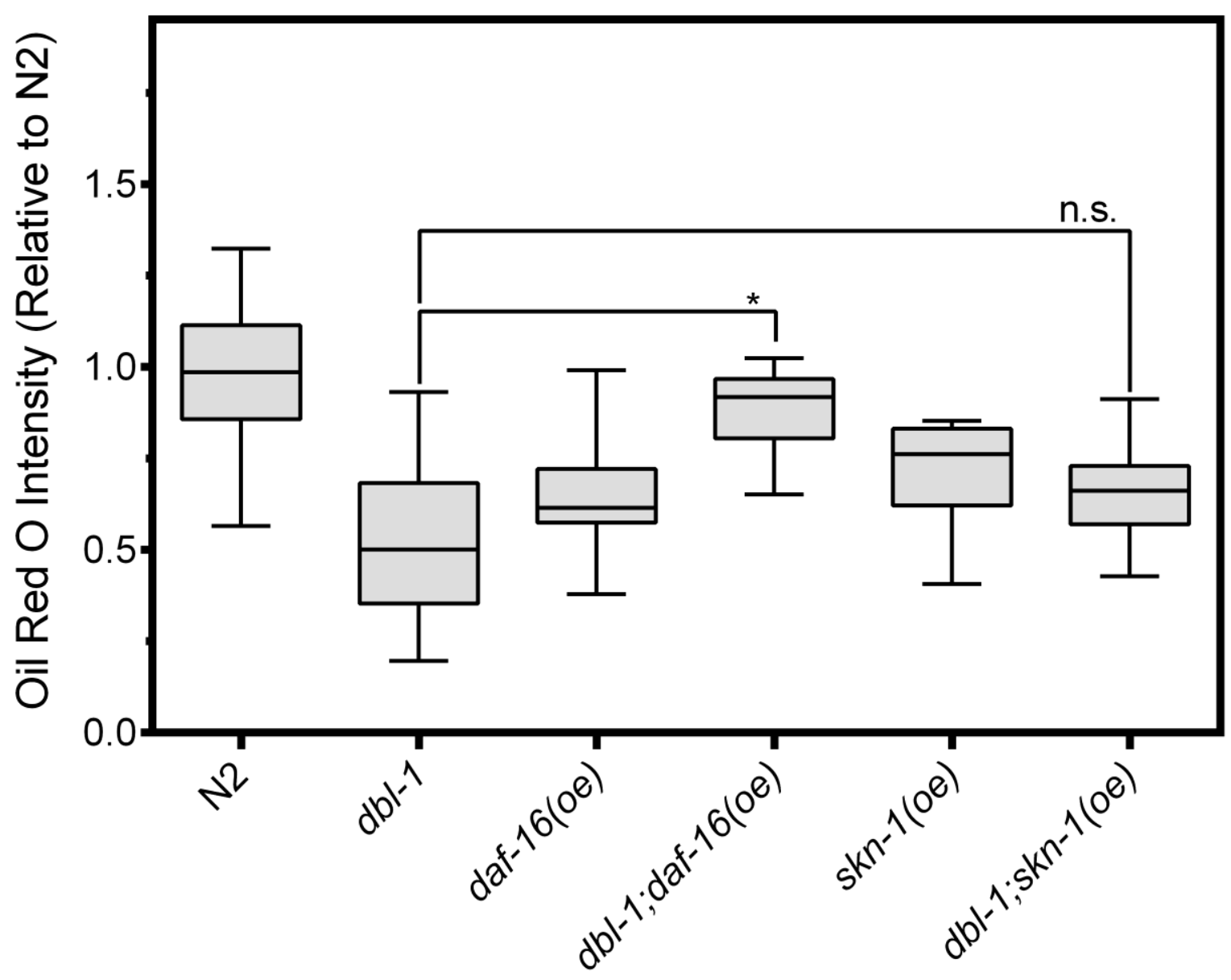

Figure 5. Effects of DAF-16/FoxO and SKN-1/Nrf overexpression on lipid accumulation.

Animals were stained at L4 stage with the neutral lipid dye Oil Red O. Images taken at 400X, camera settings were kept constant between all strains. Quantification was done for equivalent regions of the intestine just posterior to the pharynx. n.s. not significant, ${ }^{*} p$ value $<0.05,{ }^{* * *} p$ value $<0.001$, boxes denote the $2^{\text {nd }}$ and $3^{\text {rd }}$ quartiles, whiskers denote min and max values. 
A

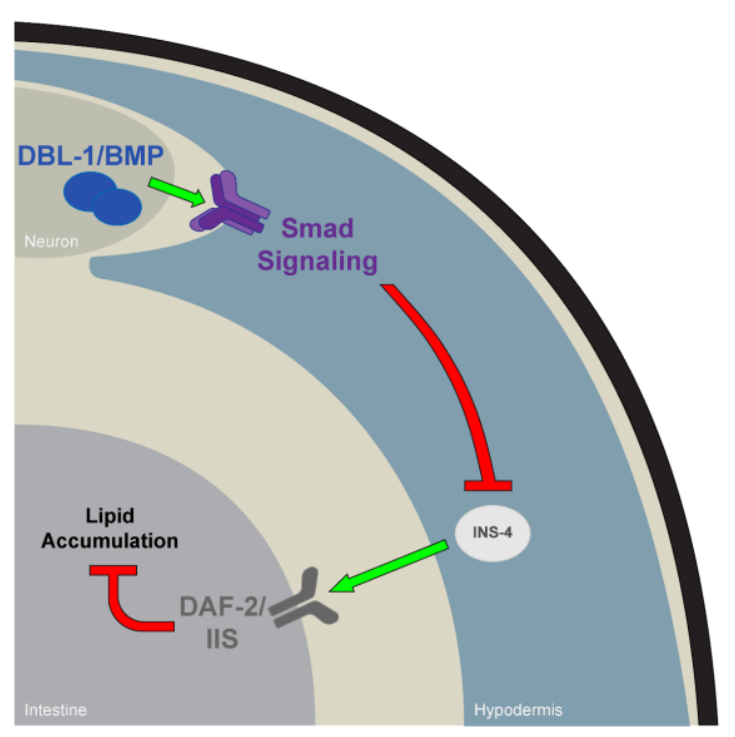

B

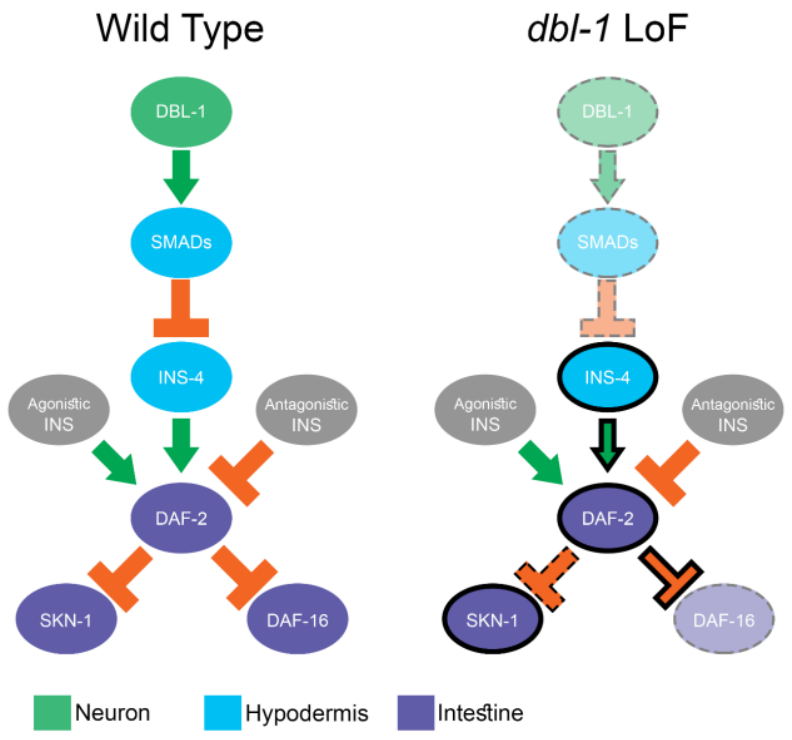

Figure 6. Model for interaction between DBL-1/BMP signaling and DAF-2/IIS pathways. A)

Smad signaling in the hypodermis regulates the transcription of ins-4, which in turn feeds into DAF-2/IIS in the intestine to control lipid metabolism. B) In wild-type animals, DAF-2/InsR is regulated by multiple agonistic and antagonistic ILPs. DAF-2/InsR activation leads to phosphorylation and cytoplasmic retention of SKN-1/Nrf and DAF-16/FoxO. In dbl-1 loss-offunction (LoF) mutants, reduced Smad activity leads to increased INS-4 expression. Also in $d b l-1$ mutants, SKN-1/Nrf nuclear localization has reduced sensitivity to DAF-2/InsR activity, while DAF-16/FoxO nuclear localization has increased sensitivity to DAF-2/InsR function. 

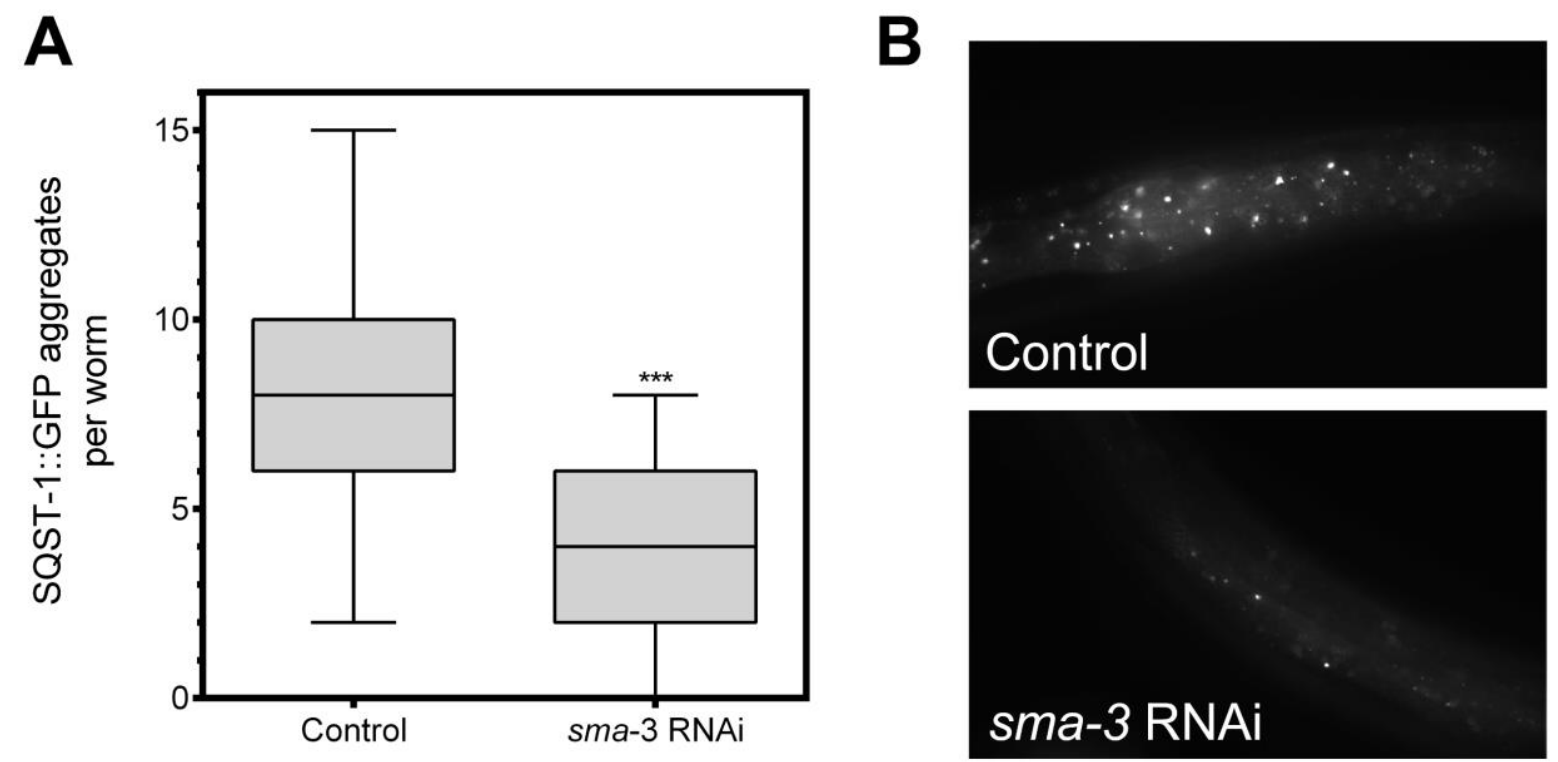

\section{S1 Fig. Inactivation of sma-3/Smad suppresses the accumulation of SQST-1::GFP}

aggregates in rpl-43 mutants. A) rpl-43 mutants show accumulation of SQST-1::GFP puncta

due to impaired autophagic flux (Guo et al. 2014). Genetic backgrounds that increase

autophagy allow the clearance of those aggregates. We analyzed this strain at the L4 stage

following RNAi depletion of sma-3/Smad compared with empty vector control (L4440), and

found a significant clearance of SQST-1::GFP puncta $(p<0.001)$ as expected (Guo et al. 2014).

Two trials of $n=15$ animals yielded similar results, and the combined data are shown. B)

Images of sqst-1::gfp animals treated with either an empty vector control (L4440) or sma-3

RNAi. 